\title{
ESR Studies of Paramagnetic Centers in Pharmaceutical Materials - Cefaclor and Clarithromycin as an Example
}

\author{
A. Skowronska ${ }^{a}$, M. Wojciechowski ${ }^{a}$, P. Ramos ${ }^{b}$, B. Pilawa ${ }^{b}$ And D. Kruk ${ }^{c, *}$ \\ ${ }^{a}$ Institute of Physics, Jagiellonian University, W.S. Reymonta 4, 30-059 Kraków, Poland \\ ${ }^{b}$ Department of Biophysics, Medical University of Silesia, Sosnowiec, Poland \\ ${ }^{c}$ University of Warmia and Mazury in Olsztyn, Faculty of Mathematics and Computer Science \\ Słoneczna 54, PL-10-710 Olsztyn, Poland
}

\begin{abstract}
Electron spin resonance (ESR) studies of two antibiotics, Cefaclor and Clarithromycin, have been performed in order to investigate concentration and dynamics of free radicals generated in these compounds due to thermal sterilization. For Cefaclor three combinations of temperature and heating time have been applied: $160{ }^{\circ} \mathrm{C}$, $170^{\circ} \mathrm{C}, 180^{\circ} \mathrm{C}$ for $120 \mathrm{~min}, 60 \mathrm{~min}$, and $30 \mathrm{~min}$, respectively, according to the pharmaceutical sterilization norm. Clarithromycin has been heated at $160^{\circ} \mathrm{C}$ for $120 \mathrm{~min}$. The ESR lineshape has been investigated versus microwave power ranging from $2.2 \mathrm{~mW}$ to $70 \mathrm{~mW}$. Electron spin-spin relaxation time has been estimated from the ESR lineshape analysis. Concentrations of radicals generated due to different sterilization procedures have been compared with the purpose to select the best sterilization scheme.
\end{abstract}

PACS: 87.15.La, 87.64.kj, 87.64.kh, 75.10.Dg

\section{Introduction}

An important part of the procedure of producing pharmaceutics is thermal sterilization. In practice this process is realized by heating the compounds at a given temperature for a given time. Typically three combinations are applied: $160^{\circ} \mathrm{C}$ for $120 \mathrm{~min}, 170^{\circ} \mathrm{C}$ for $60 \mathrm{~min}$, and $180^{\circ} \mathrm{C}$ for $30 \mathrm{~min}$. Due to the thermal sterilization free radicals are created. Performing the sterilization it is very important to keep the amount of generated radicals as low as possible because of their unwanted activity in chemical reactions. Beside the concentration of the created radicals it is also important to monitor their relaxation properties (electron spin relaxation time) that influence their effects on metabolic processes in cells.

Free radicals in drugs may be responsible for the major toxic effects in human organism. Free radicals appear in drugs as the result of physical interactions. High temperature causes paramagnetic character of drugs via homolytic reactions as the breaking of chemical bonds in their structures [1-3].

The important aim of the performed studies is to determine concentration and properties of free radicals formed in the two exemplary drugs exposed to high temperature during sterilization process. The practical purpose of this work is an optimization of the conditions of thermal sterilization for the tested drugs. The best conditions of storage are these with the lowest free radical concentration.

* corresponding author; e-mail: danuta.kruk@matman.uwm.edu.pl
This work is meant as an extension of similar studies $[2,4,5]$ performed for different antibiotics. ESR spectroscopy provides information on the relaxation dynamics of paramagnetic centers $[6,7]$ as well as on their concentration. The ESR spectrum can be treated as a probability density of the spin transition at a given frequency $\omega$ including the effect of local magnetic fields fluctuating in time (the electron spin relaxation). Thus analyzing the shape of the ESR spectrum one gets access to the electron spin relaxation. In ESR experiments first derivative of the absorption spectrum is recorded. This implies that double integral such a spectrum gives is proportional to the amount of paramagnetic centers. This implies that double integral of such a spectrum gives is proportional to the amount of paramagnetic centers. These features have been exploited to investigate the electron spin relaxation and concentration of paramagnetic centers generated due to thermal sterilization of two antibiotics: Cefaclor and Clarithromycin.

\section{Materials and methods}

Cefaclor is a semisynthetic antibiotic chemically related to penicillin, while Clarithromycin is a macrolide antibiotic. Both are used to treat bacterial infections. Their structure is shown in Fig. 1 [8]. Cefaclor has been heated in the temperature of $160^{\circ} \mathrm{C}, 170^{\circ} \mathrm{C}, 180^{\circ} \mathrm{C}$ for $120 \mathrm{~min}, 60 \mathrm{~min}$, and $30 \mathrm{~min}$, respectively, while for Clarithromycin $160^{\circ} \mathrm{C}$ has been applied for $120 \mathrm{~min}$. Then ESR spectra (X-band, $9.3 \mathrm{GHz}$ ) have been recorded versus microwave power in the range of $2.2-70 \mathrm{~mW}$ at room temperature. 

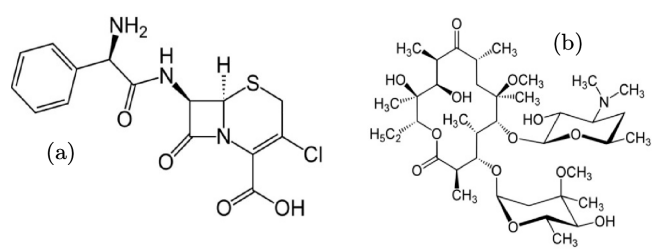

Fig. 1. Structure of Cefaclor (a) and Clarithromycin (b).

\section{Results and analysis}

The ESR spectra collected for increasing power of the microwave irradiation have been rescaled (multiplied by a constant) to reach the best possible overlapping. Then the broadening of the ESR lines with increasing microwave power is revealed. Figure 2 shows illustrative ESR spectra for Cefaclor and Clarithromycin ((a) and (b), respectively) obtained after heating these antibiotics at $160^{\circ} \mathrm{C}$ for $120 \mathrm{~min}$. The spectra can be approximated by a derivative of Lorentzian function (as it will be shown later) and they are of a rather similar width. To investigate the influence of the sterilization procedure ESR spectra for Cefaclor sterilized at the temperature of $170^{\circ} \mathrm{C}, 180^{\circ} \mathrm{C}$ for $60 \mathrm{~min}$ and $30 \mathrm{~min}$, respectively, have been collected as well; examples of them are shown in Fig. 3. For higher power of the microwave irradiation the spectra become progressively broader, however the effect is not very pronounced.
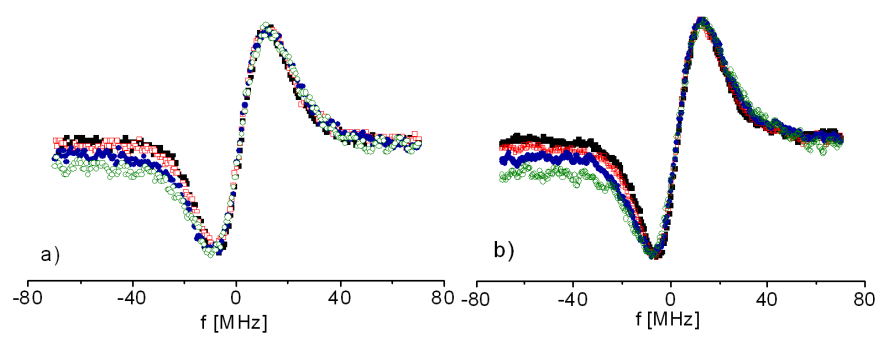

Fig. 2. Selected ESR spectra for (a) Cefaclor, (b) Clarithromycin heated at $160{ }^{\circ} \mathrm{C}$ for $120 \mathrm{~min}$ versus microwave irradiation power: $2.2 \mathrm{~mW}$ - solid squares, $7 \mathrm{~mW}$ - open squares, $17.6 \mathrm{~mW}$ - solid circles, $44.2 \mathrm{~mW}$ - open circles. The spectra have been scaled to reach the best overlapping.

The Lorentzian ESR lineshape is described (in its derivative form) as

$$
L(\Delta \omega)=A \frac{\mathrm{d}}{\mathrm{d}(\Delta \omega)}\left[\frac{\tilde{T}_{2}}{1+\left(\Delta \omega \tilde{T}_{2}\right)^{2}}\right],
$$

where $\Delta \omega=\omega-\omega_{0}$ denotes the deviation of the frequency at which the spectrum is taken $(\omega)$ from the central frequency $\omega_{0}=9.3 \mathrm{GHz} . \quad \tilde{T}_{2}$ is defined as: $\tilde{T}_{2}=T_{2} \sqrt{S}$, where $T_{2}$ is the electron spin-spin relaxation time, while $S$ is referred to as a saturation factor given as $[6,7]$ :

$$
S=\left(1+\gamma^{2} B_{1}^{2} T_{1} T_{2}\right)^{-1},
$$

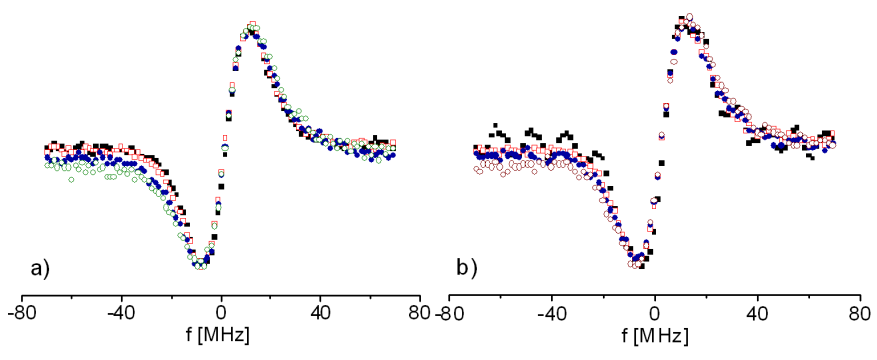

Fig. 3. Selected ESR spectra for Cefaclor thermally sterilized at (a) $170{ }^{\circ} \mathrm{C}$ for $60 \mathrm{~min}$ and (b) $180^{\circ} \mathrm{C}$ for 30 min versus microwave irradiation power: $2.2 \mathrm{~mW}-$ solid squares, $7 \mathrm{~mW}$ - open squares, $17.6 \mathrm{~mW}$ - solid circles, $44.2 \mathrm{~mW}$ - open circles. The spectra have been scaled to reach the best overlapping.

where $T_{1}$ is the electron spin-lattice relaxation time, $\gamma$ is electron gyromagnetic factor and squared alternating magnetic field $B_{1}^{2}$ is proportional to the microwave power, $P \propto B_{1}^{2}$. The values of $\tilde{T}_{2}$ versus the microwave power have been obtained for all collected spectra from least-squares fits by means of Eq. (1). Examples of such fits are shown in Fig. 4 while the obtained values of $\tilde{T}_{2}$ are displayed in Fig. 5 versus the microwave irradiation power.

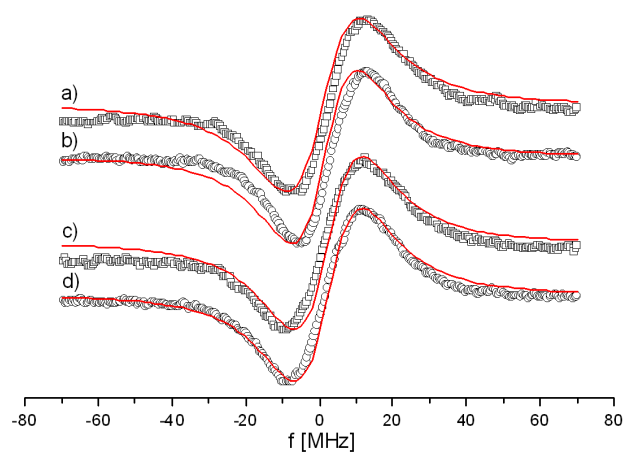

Fig. 4. ESR spectra for (a) Cefaclor, $8.8 \mathrm{~mW}, 160{ }^{\circ} \mathrm{C}$, $120 \mathrm{~min}$, (b) Cefaclor, $31.5 \mathrm{~mW}, 160^{\circ} \mathrm{C}, 120 \mathrm{~min},(\mathrm{c})$ Clarithromycine, $8.8 \mathrm{~mW}, 160^{\circ} \mathrm{C}, 120 \mathrm{~min}$, (d) Clarithromycine, $31.5 \mathrm{~mW}, 160^{\circ} \mathrm{C}, 120 \mathrm{~min}$. Solid lines corresponding Lorentzian fits.

The spin-spin relaxation time $T_{2}$ can be obtained from the analysis of the ESR lineshape for low radiofrequency power. In this case one can approximate them by the values at $2.2 \mathrm{~mW}$ which are equal to $11.2 \mathrm{~ns}, 11.7 \mathrm{~ns}, 12.1 \mathrm{~ns}$ and $11.4 \mathrm{~ns}$ for Cefaclor, $160^{\circ} \mathrm{C}, 170^{\circ} \mathrm{C}, 180^{\circ} \mathrm{C}$ and Clarithromycine, $160^{\circ} \mathrm{C}$, respectively. These values do not differ significantly. Taking into account that $P \propto B_{1}^{2}$ one can write the power dependence of the effective relaxation time $\tilde{T}_{2}$ as $[6,7]$ :

$$
\tilde{T}_{2}=\frac{T_{2}}{\sqrt{1+\xi P T_{2}}} .
$$

The parameter $\xi$ is given as $\xi=\frac{B_{1}^{2}}{P} \gamma^{2} T_{1}$ where the ratio $\frac{B_{1}^{2}}{P}=$ const is a spectrometer constant. The $\xi$ values 


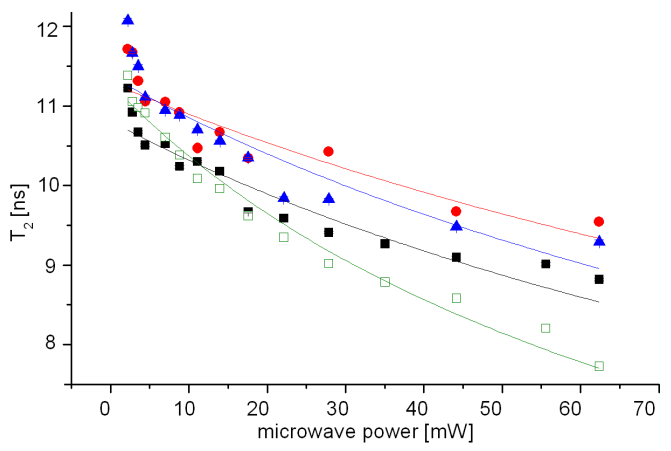

Fig. 5. $\tilde{T}_{2}$ versus microwave irradiation power for Cefaclor, $160^{\circ} \mathrm{C}$ - solid squares; Cefaclor, $170{ }^{\circ} \mathrm{C}-$ solid circles; Cefaclor, $180^{\circ} \mathrm{C}$ - solid triangles; Clarithromycine, $160^{\circ} \mathrm{C}$ - open squares. Corresponding solid lines - fits according to Eq. (3).

obtained for Cefaclor, $160^{\circ} \mathrm{C}, 170{ }^{\circ} \mathrm{C}, 180^{\circ} \mathrm{C}$ and Clarithromycine, $160^{\circ} \mathrm{C}$ are respectively equal to $(8.9 ; 6.5$; 8.6 and 16.3$) \times 10^{8} \mathrm{~J}^{-1}$. These values indicate that the spin-lattice electron spin relaxation time for radicals in Clarithromycine is about twice longer than for Cefaclor. To get insight into the electron spin relaxation it is also interesting to analyze the amplitude of the ESR spectrum as a function of the applied microwave power. The amplitude has been taken as an average of the amplitudes of the minimum and maximum, $A=\left(A_{\min }+A_{\max }\right) / 2$ and normalized to one for the highest amplitude keeping the ratios between values.

The experimental dependencies are shown in Fig. 6 . For Lorentzian lines the amplitude is described by the relationship

$$
A \propto \gamma^{2} T_{2}^{2} P^{1 / 2} S^{3 / 2}=\gamma^{2} T_{2}^{2} P^{\frac{1}{2}}\left(1+\xi P T_{2}\right)^{-3 / 2},
$$

where the definition of the saturation factor $S$ of Eq. (2) has been substituted. This means that for small microwave powers the amplitude increases with $\sqrt{P}$, while for larger powers it decreases linearly with $P$ (eventually a saturation is reached that is not predicted by Eq. (4) but observed in Fig. 6). In this figure one sees the linear decrease of the amplitude with the microwave power indicated by solid straight lines. The slopes of the decays are similar for Cefaclor (independent of the sterilization procedure), but different for Clarithromycine, again indicating somewhat different spin-lattice relaxation of the paramagnetic centers in both compounds.

Summarizing this part of the work one observes that the electron spin relaxation does not differ much for different sterilization procedures; one can see this explicitly from Fig. 3 in which rescaled ESR spectra for Cefaclor that underwent sterilization at $160^{\circ} \mathrm{C}, 170^{\circ} \mathrm{C}, 180^{\circ} \mathrm{C}$ for $120 \mathrm{~min}, 60 \mathrm{~min}$, and $30 \mathrm{~min}$, respectively, are directly compared for a selected microwave irradiation power. One can also conclude at this stage that the relaxation dynamics of the paramagnetic centers is very similar for both compounds Cefaclor and Clarithromycin.

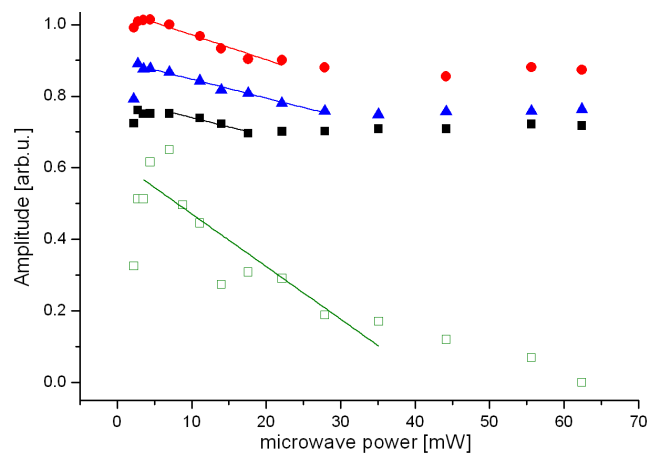

Fig. 6. Amplitude of ESR spectra for Cefaclor, $160^{\circ} \mathrm{C}$ — solid squares; Cefaclor, $170^{\circ} \mathrm{C}$ - solid circles; Cefaclor, $180^{\circ} \mathrm{C}-$ solid triangles, and Clarithromycine, $160^{\circ} \mathrm{C}$ - open squares.

As long as we consider normalized spectra with the purpose to analyze their shape and determine the relaxation time, the amplitude of the lines is irrelevant. It is however of importance when one discusses the concentration of the radicals created in the sample. Assuming that all spectra are of the same shape (they are Lorentzian in this case) and that the same amount of material has been taken the amplitude gives directly the relative concentration of radicals, i.e. the ratio of amplitudes determines the ratio of concentrations. In order to estimate the relative concentration of the created radicals the amplitudes of nonscaled spectra (but taken under the same condition and for the same amount of material) have to be compared as shown in Fig. 7.

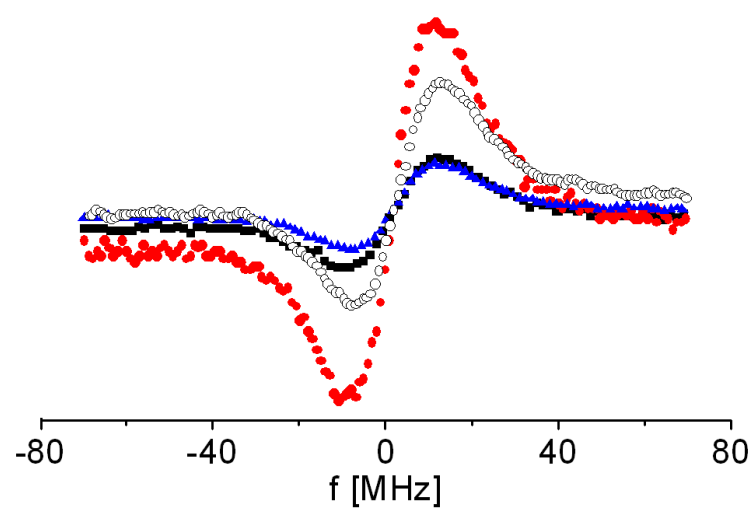

Fig. 7. Non-scaled ESR spectra for Cefaclor sterilized at $160^{\circ} \mathrm{C}, 120 \mathrm{~min}$ (solid squares); $170^{\circ} \mathrm{C}, 60 \mathrm{~min}$ (solid circles); $180^{\circ} \mathrm{C}, 30 \mathrm{~min}$ (solid triangles); and Clarithromycine, $160^{\circ} \mathrm{C}, 120 \mathrm{~min}$ (open circles) $(P=$ $55.60 \mathrm{~mW})$. The same mass of the material has been used.

One can see from this comparison that the thermal sterilization of Cefaclor should not be performed at the intermediate temperature $\left(170^{\circ} \mathrm{C}\right)$ for the intermediate time (60 $\mathrm{min})$ because then the concentration of the generated paramagnetic centers (radicals) is $4-5$ times larger 
than for the other combinations of time and temperature. The situation can be different for other compounds then another sterilization procedure can turn out to be the most appropriate one. The concentration of radicals for different pharmaceutics that underwent the same sterilization procedure can also vary. Comparing the case of Clarithromycine and Cefaclor (both sterilized at $160^{\circ} \mathrm{C}$ for $120 \mathrm{~min}$ ) one can see that in the first case the concentration of radicals is considerably lower. These results are of high practical application for pharmaceutical industry.

\section{Conclusions}

ESR spectroscopy of thermally sterilized antibiotics, Cefaclor and Clarithromycine, have been performed in order to get information on the concentration of paramagnetic centers generated due to different protocols of the sterilization procedure. It has turned out that while the relaxation features of the centers are similar for both compounds, they are not considerably affected by the sterilization procedure. The electron spin-spin relaxation time has been estimated from ESR spectral analysis for low radiofrequency power. It has also been shown that the concentration of the paramagnetic centers is, however, strongly dependent on the details of sterilization.

\section{Acknowledgments}

This work was supported by funds for science in years 2009-2012 as research project No. N N202 105936 (Polish Ministry of Science and Education).

\section{References}

[1] P. Ramos, B. Pilawa, S. Wilczyński, K. Czyż, J. Adamczyk, Eng. Biomat. 12, 7 (2009).

[2] P. Ramos, B. Pilawa, Curr. Top. Biophys. 33, suppl. A, 183 (2010).

[3] P. Ramos, B. Pilawa, M. Biernaś, J. Med. Inf. Technol. 15, 185 (2010).

[4] P. Ramos, B. Pilawa, Curr. Top. Biophys. 33, suppl. B, 47 (2010).

[5] P. Ramos, P. Peplonski, B. Pilawa, Eng. Biomat. 12, 162 (2009).

[6] A. Abragam, B. Bleaney, Electron Paramagnetic Resonance of Transition Ions, Dover Publications, New York 1986.

[7] J.E. Wertz, J.R. Bolton, Electron Spin Resonance. Theory and Practical Application, Chapman and Hall, London 1986.

[8] A. Zejca, Chemia leków (Chemistry of Medicines), PZWL, Warszawa 2004 (in Polish). 